\title{
7. Composition as General Identity
}

\author{
Aaron J. Cotnoir
}

What is the relationship between a whole and its parts taken together? David Lewis famously claimed, 'They are it. It is them' $(1991,83)$. In almost the next breath, however, he retracts his claim and 'rests content with mere analogy' (1991, 84, fn. 12). Why did Lewis reject the strong claim that composition just is identity in favor of the weaker claim that composition is a lot like identity? ${ }^{1}$

He did so because, like many others, he could not make sense of strong composition as identity. In particular, he had two main obstacles. He writes:

In the first place, I know of no way to generalize the definition of ordinary one-one identity in terms of plural quantification. (87)

In other words, he could see no good way of making sense of manyone identity. If we cannot make sense of many-one identity, and the parts are many while the whole is one, then it appears that composition as identity is unintelligible. Moreover, Lewis suggests:

And in the second place,... still we do not really have a generalized principle of the indiscernibility of identicals. It does matter how you slice it...What's true of the many is not exactly what is true of the one. (87)

The whole may have properties lacked by its parts taken together; the parts may have properties lacked by the whole. And the failure of the indiscernibility of identicals leads us to believe that the relation between a whole and its parts is not identity after all.

The aim of this chapter is to formalize (a version of) composition as identity, addressing these obstacles. In section 1, I address a challenge present in Lewis but made most explicit by van Inwagen (1994) — the SYNTACTIC CHALLENGE — by appealing to free relatives in English as operators that shift syntactic number while leaving

${ }^{1}$ I will follow Yi's (1999) usage of strong composition as identity for the identity claim, and weak composition as identity for the 'a lot like identity' claim. 
semantic number fixed. In section 2, I respond to Lewis's first obstacle: the SEMANTIC CHALLENGE. I start by giving a heuristic set-theoretic model of one interpretation of many-one identity, and then show how this identity relates to other standard notions like plural identity and parthood. In section 3, I address Lewis's second obstacle: the DISCERNIBILITY CHALLENGE. I utilize two cover-based semantics for collective, distributive, and cumulative predication in plural logics that require no restrictions on the indiscernibility of identicals. This shows that the general identity relation given in section 3 is a genuine form of identity. In section 4, I show how this theory avoids recent arguments from Sider (2007). The result is an intelligible version of the thesis that composition is identity. I close with an appendix providing technical results.

\section{SYNTAX, SEMANTICS, AND IDENTITY}

Those who endorse strong composition as identity commit to the following two identity claims: ${ }^{2}$

(1) The fusion of them is them

(2) They ${ }_{i}$ are the fusion of them $i$

Lewis's first challenge is to make sense of the one-many and manyone identity relations expressed in (1) and (2). ${ }^{3}$ But it is unclear how to make sense of such a hybrid 'is/are' identity predicate in English.

The standard distinction between the syntax of a language and the semantics of a language reveals that there are really two challenges present here. Let us label them as follows.

SYNTACTIC CHALLENGE Is there any way to make syntactic sense of an identity predicate that takes a plural term and a singular term in each of its argument places?

SEMANTIC CHALLENGE Is there any way to make semantic sense of an identity relation such that many things are identical to one thing?

The first question is fundamentally about the structure of language while the second is primarily about the referents of terms and the

\footnotetext{
2 See Lewis (1991). Indexes will be used to facilitate cross reference.

3 The concept of 'many-one identity' is due to Don Baxter (1988).
} 


\section{6 | Aaron J. Cotnoir}

relations between those entities. The one is a question for linguists, the other for metaphysicians.

But philosophers have been concerned along syntactic lines. van Inwagen (1994) has argued that strong composition as identity theorists cannot use English to grammatically state their own view.

There is the 'is' of (singular) identity. This word makes syntactical sense when it is flanked by singular terms and variables... There is the 'are' of (plural) identity. This word makes sense when it is flanked by plural terms and plural variables... But what kind of syntactical sense is there in taking either the 'is' or 'are' and putting a singular term or variable on one side of it and a plural term or variable on the other? (210-11)

The thrust of the objection is that such sentences are syntactically unacceptable in English. Sider (2007) flags the sYNTACTIC CHALLENGE as well.

But now there is a problem. Given the truth of 'Ted $=h, a, l$, and $t^{\prime}$ and 'Ted is human', [Leibniz's] Law apparently implies that ' $h, a, l$, and $t$ is human' is true. This sentence is ungrammatical; the logic/English hybrid we are now speaking at best allows ' $h, a, l$, and $t$ are human'... Grammatical revisionism was perhaps already in place right at the start. (57)

Sider's response to the concern is to move to a formal language without the singular-plural distinction-in effect, a one-sorted plural first-order language. I, with Sider, am happy to regiment the theory in a formal language that allows for such peculiarities.

It is worth noting, however, that whether van Inwagen's and Sider's claims are correct depends heavily on the results of the best theories of agreement in the syntax of English. Thus, the SYNTACTIC CHALLENGE strikes me as an empirical question for linguists to answer, not a challenge that can be leveled from the armchair. Moreover, it is unclear why-apart from linguistic prejudice-we should only be concerned with the syntactic constraints of English. In other natural languages (e.g. Nordic languages), the construction is perfectly syntactically acceptable. For Norwegian, the verb meaning to be ('være') is conjugated the same (present tense: 'er') for both singular and plural noun phrases. ${ }^{4}$

But let's not dismiss the syntactic CHALLENGE so quickly. As it turns out, some linguists and philosophers of language think that such semantically hybrid constructions exist in English, too.

${ }^{4}$ Thanks to Einar Bøhn, Jon Litland, and Andreas Fjellstad here. 


\subsection{Singular, plural, many, one}

Consider the following two distinctions: syntactically plural vs syntactically singular, and semantically plural vs semantically singular.

An English term is syntactically plural if it is morphologically plural, and syntactically singular if it is morphologically singular. The syntactic difference between singular and plural morphemes often takes the form of different endings (e.g. 'fungus'; 'fungi') and usually requires different verb conjugation (i.e. 'The dog barks.'; 'The dogs bark.'). The basic idea is to take singular and plural morphemes of the same lexeme; but the idea extends to pronouns and other noun phrases as well (e.g. 'it'; 'them').

Also, let a term be semantically plural if the referent of the term is more than one object, and let it be semantically singular if the referent is only one object. Of course, the main topic of this chapter is tied up with controversies over what counts as one object and what counts as more than one object. Needless to say, whether a given term is semantically plural or singular may be controversial. It may even be controversial as to whether there is any mind-independent fact of the matter as to whether the referent of a term is many or one.

It might come as a surprise if syntactic number and semantic number were perfectly aligned. Unsurprisingly, the distinctions cut across each other. We can find examples-perhaps not uncontroversial ones - of syntactically plural terms that are semantically singular. Consider 'scissors' and 'pants'. Both have morphologically plural endings, conjugate plurally ('The scissors are sharp.'; *'The scissors is sharp.'), and yet putatively refer to a single thing. ${ }^{5}$ It may be noted that 'scissors' and 'pants' are linked to constructions like 'pair of scissors' and 'pair of pants', which are syntactically singular. But this simply reinforces the conclusion that, in English, we may use syntactically singular and plural constructions to refer to the very same thing(s).

Likewise, we can find examples of syntactically singular terms that are semantically plural. Consider 'clothing', 'furniture', and 'jury'. These terms are morphologically singular, conjugate singularly ('The furniture is Federal.'; *‘The furniture are Federal.'), and yet each of them refer to many things. ${ }^{6}$

\footnotetext{
5 Thanks to Leo Iacono for suggestions here.

${ }^{6}$ But see Laycock (2006) for a view according to which some of these terms refer neither singularly, nor plurally. See also Uzquiano (2007) for a view according to which some of these terms are semantically singular.
} 


\section{8 | Aaron J. Cotnoir}

The fact that these distinctions cut across each other may be displayed in other ways. In British English, verb conjugation is sometimes keyed to semantic number rather than syntactic number. Consider the headlines 'Arsenal win by one' or 'Parliament disagree on proposal'. Even American academics tend to find both of the following sentences syntactically acceptable: 'The faculty try hard not to fight.' and 'The faculty tries hard not to fight.' Likewise, when asked 'Where is the jury?', it would appear perfectly acceptable to respond 'They are still deliberating'.

If these examples are unconvincing, the reader is welcome to think of others. The idea that semantic number and syntactic number have some independence from one another does not by itself address the SYNTACTIC CHALLENGE to composition as identity. However, this fact, combined with an additional claim does address the objection. The claim: English has the resources for syntactic singularization of semantically plural terms.

\subsection{Singularization in natural language}

I do not intend to undertake a full defence of this claim here. I take this to be an empirical question, not suitably answered by philosophical reflection. However, I will say a few things by way of explanation and point to some linguistic evidence.

The rough idea is that English speakers have the linguistic resources to singularly refer to many things whenever they can plurally refer to those things. Phillipe Schlenker (MS) has argued that even though plurals are explicitly morphologically typed, they are badly typed. He suggests that a certain kind of definite description-called a free relative-behaves as a type-shifting mechanism transforming plurals into singular terms.

A natural suggestion, then, is that what-descriptions provide the missing link because they are... morphologically singular and yet, in a sense, semantically plural. (11)

Consider Schlenker's example of a what-description compared to standard definite description.

\footnotetext{
7 Thanks to Sam Wheeler, John Troyer, and Marcus Rossberg for suggestions here.
} 
(3) I bought some things. The things I bought *was/were expensive.

(4) I bought some things. What I bought was $/{ }^{*}$ were expensive.

Contrary to usual definite descriptions, the what-description triggers singular verb agreement. ${ }^{8}$ But the referent of 'what I bought' is not one of the things. After all, each of the things may be relatively inexpensive individually but collectively expensive nonetheless. The referent is not the set of things; sets are abstracta and are not expensive. Given the appearance that (3) and (4) have the same semantic content, Schlenker plausibly suggests that the referent of 'what I bought' is the things themselves.

Oliver and Smiley (2008) also address free relatives from a plural perspective. They independently note the same phenomenon. ${ }^{9}$ Consider one of their examples:

'I took what they offered me' is tantamount to 'I took the things they offered

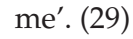

Here they appeal to the fact that sentences involving free relatives and parallel sentences involving plurals have the same semantic content.

Now, finally, we can substantiate the claim that English has resources to singularize syntactically plural terms while maintaining plural reference. ${ }^{10}$

SINGULARIZATION For any syntactically plural term $x x$, there is some syntactically singular term 'what they ${ }_{x x}$ are identical to' such that the referent of $x x$ is the same as the referent of 'what they $y_{x x}$ are identical to'.

SINGULARIZATION is the conclusion of some linguists. As mentioned, the claim of SINGULARIZATION with respect to the resources of

8 The what-description is not the standard definite description operator; nor is it a plural definite description operator. See Rayo $(2002,446)$ for a definition of plural definite descriptions; or for a more general account, see Sharvey (1980).

9 They give three readings of free relatives, and each reading displays the same phenomenon of syntactic singularization. The example that follows is intended as an example of the definite reading.

${ }^{10}$ It should be noted that, when combined with unrestricted comprehension for plural terms, SINGULARIZATION is inconsistent in classical logic. See Schlenker (MS, 10). But plural comprehension will already have to be restricted for composition as identity theorists, due to conflicts with Cantor's theorem. It is an interesting additional question as to whether more restrictive versions of plural comprehension combined with SINGULARIZATION are classically consistent. One version is given in the Appendix 1. For more discussion, see Saucedo (Unpublished). 
English is ultimately empirical. But if correct, it can be used to formulate a syntactically unobjectionable version of the one-many identity claim in (1) and the many-one identity claim in (2).

(5) The fusion of them is what they $y_{i}$ are identical to.

(6) What they $y_{i}$ are identical to is the fusion of them

Given Singularization, 'what they are identical to' is a syntactically singular term with semantically plural reference. 'The fusion of them,' is both syntactically and semantically singular. So, what we have, from a syntactic point of view, is a singular-singular identity statement. There is no grammatical abnormality. From a semantic point of view, however, we have a full-blown many-one identity claim.

This response to the sYNTACTIC CHALLENGE is dependent on a theory of plurals that has not been given a full defence. Nonetheless, I have highlighted a (possibly true) linguistic theory according to which many-one identity claims are perfectly grammatical in English.

\section{MANY-ONE IDENTITY}

We now turn in earnest to the SEMANTIC CHALLENGE that concerned Lewis. He writes:

I know of no way to generalize the definition of ordinary one-one identity in terms of plural quantification. We know that $x$ and $y$ are identical iff, whenever there are some things, $x$ is one of them iff $y$ is one of them. But if $y$ is the fusion of the $x x$, then there are some things such that each of the $x x$ is one of them and $y$ is not; and there are some things such that $y$ is one of them but none of the $x x$ is. $(1991,87)$

Lewis is here registering the complaint that the ordinary plural definition of identity in terms of the is-one-of relation does not extend to a many-one identity relation. On the standard semantics of identity, a singular (one-one) identity claim like ' $a=b$ ' is true if and only if ' $a$ ' and ' $b$ ' denote the same object. Plural (many-many) identity can be defined in terms of the 'is-one-of' predicate, thus: ' $a a=b b$ ' is true if and only if for all $x, x$ is one of $a a$ if and only if $x$ is one of $b b$. Intuitively, an identity claim is true exactly if the terms on either side of the identity symbol denote the same object(s). How can this be generalized to many-one identity? 
As should be apparent, the standard treatment of identity as sameness of reference will not work. Take a many-one identity claim like ' $a a=b$ '. If ' $a a^{\prime}$ is a semantically plural term, its referent will be many things; and in order for the identity to hold, the referent of ' $b$ ' would have to be those same things. But that contradicts the supposition that ' $b$ ' is semantically singular. If the discussion in section 1.2 is correct, then ' $b$ ' may be semantically plural even if syntactically singular, but even then this will not be a satisfactory solution to the semantic problem.

So we need some other semantic treatment of many-one identity, preferably one that describes some more general phenomenon that has many-many and one-one identity as a special case. For this task, I construct a set-theoretic interpretation of a generalized identity relation that gives the right results. I do not, however, mean to be assuming the ontology of the semantics. For those with ontological qualms about using set theory for semantics, the semantics could be done using hyperplurals; but given the relative unfamiliarity with higherorder plural quantification, the set-theoretic interpretation will serve as a useful guide. ${ }^{11}$ The reader should beware of reading additional ontological commitments into the set-theoretic discussion.

In order to take many-one identity seriously, we need to suppose that we can refer to a portion of the world singularly or plurally, and that our way of referring to this portion of the world does not change the fact that it is the same portion either way. The intuitive pull behind composition as identity is the thought that we may 'carve up' reality however we like. But no matter whether we carve a portion of it as one individual or many, it is still the same bit of reality. Compare Lewis (1991):

Take them together or take them separately, [they] are the same portion of reality either way. (81)

And recall Frege, who in the Foundations of Arithmetic (1980) claimed:

If, in looking at the same external phenomenon, I can say with equal truth 'This is a copse' and 'These are five trees', or 'Here are four companies' and 'Here are five hundred men', then what changes here is neither the individual nor the whole, the aggregate, but rather my terminology. (Section 46)

${ }^{11}$ For a semantics of hyperplurals that could be adapted for this purpose, see Rayo (2006). 
They are 'the same external phenomenon'; the five trees are the copse. What we want, then, is a way of modeling the concept of being 'the same external phenomenon' via ways of carving up the world.

To model this, we start with a set $A$ of objects. You may think of them as atoms. ${ }^{12}$ Recall, the intuitive idea behind many-one identity is that identities are insensitive to our ways of counting things. In other words, what there is, and hence what is generally identical to what, does not depend on our practices of counting. A simple way of modeling this phenomenon is to look at all partitions of $A$.

PARTITION A set $P$ of non-empty sets is a partition of $A$ if: (i) $P$ covers all of $A$ (i.e. $\cap P=A$ ); and (ii) the members of $P$ are pairwise disjoint (i.e. $p_{i} \cap p_{j}=\varnothing$ for any $p_{i}$ and $p_{j}$ in $P$ s.t. $\left.i \neq j\right)$.

Partitions are essentially ways of counting a domain. ${ }^{13}$ Condition (i) guarantees that in any count every portion of reality gets included in the count (even if not as a single thing). Condition (ii) rules out counting the same external phenomenon more than once; it is a prohibition against 'double counting'. ${ }^{14}$

How is this useful? A partition carves up reality into chunks or portions; it treats all the atoms in the same portion as a single thing. Partitioning a domain is a way of carving up reality into individual objects-a 'way of counting'. Different partitions might carve up a subset of $A$ as one chunk, or many chunks. But whether treated as one or many, it is still the same subset of $A$-it is still the same underlying portion of reality.

We can express a relation on $A$ via a binary predicate $\sim$. Where $x$ and $y$ are singular terms (or variables), $\bar{x}$ is the denotation of $x$, and $\pi$ is any partition on $A$ :

${ }_{12}$ You may think of them as atoms, but one needn't think of them as atoms. (Whether composition as identity is committed to atomism is an interesting question, but beyond the scope of this chapter.) Another possible interpretation-to be explored in future work-is to think of them merely as propertied spacetime points. I largely leave the underlying metaphysics open, since the semantic approach endorsed here is compatible with a number of metaphysical views.

${ }^{13}$ They are, roughly, a formalization of Baxter's ways of counting. See (1988).

${ }^{14}$ For justification of the 'no double counting' view of ontological commitments, see Baxter (1988) and Varzi (2000). See also the discussion in what follows. 
EQUIV $x \sim y$ is true iff $\bar{x}$ is in $\pi$ and $\bar{y}$ is in $\pi$.

The predicate $\sim$ expresses (roughly) the relation of being in the same count; that is, it is true of some things whenever there is a way of counting the world that includes both objects. As a consequence of condition (ii), some objects can never be in the same count. Keeping the same intended meaning, we can generalize $\sim$ to take plural terms on either side as well.

We now let singular terms $x, y, z$ and plural terms $x x, y y, z z$ range over members of partitions on $A$. So, a singular term $x$ has a denotation $\bar{x}$ that is a chunk of atoms. A plural term $x x$ has a denotation $\overline{x x}$ that is a set of chunks of atoms under the same partition. That is, we do not allow a given plural term to simultaneous refer to members of different partitions; $x x$ must always refer under the same 'way of counting' ${ }^{15}$

We now have the tools to give a heuristic account of a generalized identity predicate, $\approx .{ }^{16}$ Where $\overline{x x}$ is a subset of partition $\pi$ on $A$ and $\overline{y y}$ is a subset of a (possibly distinct) partition $\pi^{\prime}$ on $A$,

$$
\begin{aligned}
& \text { MANY-MANY } x x \approx y y \text { is true iff } \bigcup \overline{x x}=\bigcup \overline{y y} \\
& \text { MANY-ONE } x x \approx y \text { is true iff } \bigcup \overline{x x}=\bar{y} \\
& \text { ONE-MANY } x \approx y y \text { is true iff } \bar{x}=\bigcup \overline{y y} \\
& \text { ONE-ONE } x \approx y \text { is true iff } \bar{x}=\bar{y}
\end{aligned}
$$

Now, $\approx$ is a general notion of identity that holds between portions of reality independent of our ways of counting it. ${ }^{17}$ In short, it does not matter how we partition the domain, whether we count it as one or many, it is still the same portion either way.

15 This restriction amounts to a restriction on plural comprehension, and is necessary for cardinality reasons. Moreover, see the previous discussion of plural comprehension in footnote 10 .

${ }^{16}$ For ease of explication, I will call this notion 'general identity' in what follows. Whether this is really a kind of identity will be addressed in section 3. I will use the term 'numerical identity' to refer to the standard singular and plural notions.

${ }^{17}$ Indeed, set union $\cap$ is used to collapse the partitioned subset into a subset of atoms. Alternatively, one could use mereological fusion; however, doing so would be question-begging on a number of grounds. One major reason is that set union is unrestricted, and I do not want to suppose that composition as identity is committed to unrestricted mereological composition. On a hyperplural semantics neither set union nor fusion operators of any kind are required. 


\section{4 | Aaron J. Cotnoir}

It is worth noting that the MANY-MANY version of $\approx$ is not the standard notion of plural identity as defined by $a a=b b$ iff for every $x, x$ is one of the $a a$ iff $x$ is one of $b b$. There may be two ways of counting a portion of reality as many. Consider, for example, a black-and-white tile floor patterned in such a way that tiles are grouped into larger squares of the same colour. On one way of counting, there are just the many tiles; on another, there are the black squares and the white squares; on a third way of counting, there is just the tile floor. The first two ways of counting treat the floor as many. They are different partitions in the sense that the tile count is finer than the square count. But the many tiles are identical to the many squares. Notice, however, that it simply is not the case that for any $x, x$ is one of the tiles if and only if $x$ is one of the squares. In fact, none of the tiles are among the squares, and none of the squares are among the tiles.

However, the MANY-MANY version of $\approx$ is not inconsistent with ordinary plural identity. In fact, the MANY-MANY version just is ordinary plural identity whenever $x x$ and $y y$ refer to members of the same partition. In other words, we can recover ordinary plural identity via the following definition.

$$
\text { PLURAL IDENTITY } \quad x x=y y \equiv_{d f} x x \sim y y \wedge x x \approx y y
$$

Thus, MANY-MANY may be a relation between objects in different counts-a cross-count identity relation-or a relation between objects within a count-an intra-count identity relation. ${ }^{18}$ If MANYMANY is intra-count, it is just the standard plural identity relation.

By contrast, the true MANY-ONE and ONE-MANY identity claims are always cross-count. The true ONE-ONE identities are always intracount, and in this case amount to just the standard first-order identities. $^{19}$

General identity given by $\approx$ can be generalized further.

ARE-COVERED-BY $x x \lesssim y y$ is true iff $\bigcup \overline{x x} \subseteq \overline{y y}$

ARE-PARTS-OF $x x \lesssim y$ is true iff $\bigcup \overline{x x} \subseteq \overline{y y}$

${ }_{18}$ Again, these distinctions were first made by Don Baxter (1988).

${ }_{19}$ This is a slight simplification. Both one-one and many-many identities can be cross-count whenever the objects denoted are in the intersection of the two partitions. This is reflected in the full semantics in Appendix 1. 
IS-COVERED-BY $x \lesssim y$ is true iff $\bar{x} \subseteq \bigcup \overline{y y}$

IS-PART-OF $x \lesssim y y$ is true iff $\bar{x} \subseteq \bigcup \overline{y y}$

The connection between $\lesssim$ and $\approx$ can be exploited yielding the following definition: $x x \approx y y \equiv_{\mathrm{df}} x x \precsim y y \wedge y y \precsim x x$. Parallel definitions hold for the MANY-ONE, ONE-MANY, and ONE-ONE cases.

Again, and for similar reasons, ARE-COVERED-BY is not the same relation as the standard plural relation 'are-among'; it is more like the relation 'help compose'. When $x x$ and $y y$ are interpreted by the same partition, however, the difference collapses. The standard plural 'are-among' relation is intra-count, whereas $\lesssim$ may be either cross-count or intra-count. Likewise, Is-COVERED-BY reduces to the standard plural 'is-one-of' relation only if $x$ and $y y$ are interpreted by the same partition. If not, then it is more like the relation 'helps compose'. Again, the standard notions can be recovered in a straightforward way:

PLURAL ARE-AMONG $x x \leq y y \equiv_{\mathrm{df}} x x \sim y y \wedge x x \lesssim y y$

PLURAL IS-ONE-OF $x \preceq y y \equiv_{\mathrm{df}} x \sim y y \wedge x \lesssim y y$

These relations behave exactly like the plural logic versions. Plurals are extensional in the sense that $z \preceq x x$ iff $z \preceq y y$ implies $x x=$ $y y$, where $=$ here is the intra-count plural identity relation defined earlier. ${ }^{20}$ Plurals are also comprehensive in the sense that every subset of our set of atoms $A$ is denoted by some plural term. Moreover, a stronger result holds: for every possible partition on $A$, there is a plural term that denotes each subset of that partition. ${ }^{21}$

The composition as identity theorist can also recover other standard notions. IS-PART-OF is very much like the standard mereological parthood relation. Recall that a partition is simply a way of counting. If we are not picky about ways of counting-that is, if we allow all possible partitions as admissible ways of countingthen the one-one version of $\lesssim$ satisfies all the axioms of atomic

${ }^{20}$ This is a consequence of the extensionality of sets used in the model theory. See Appendix 2.

${ }_{21}$ Again, this is a consequence of the model theory; see Appendix 1. They are not, however, comprehensive in the sense that every subset of the domain of all objects (e.g. all things referred to by a term) is represented by a plural term. Again, this is mainly for cardinality reasons involving Cantor's theorem. See footnotes 11 and 16. 


\section{6 | Aaron J. Cotnoir}

classical extensional mereology. ${ }^{22}$ The most detailed way of counting-the finest partition-yields all the atoms; the least detailed way of counting - the coarsest partition-yields the whole universe. Intermediary ways of counting may be thought of as different 'levels of decomposition'.

If we have a name for the whole universe in our language (say, $\top$ ), we have a straightforward way of determining whether a plurality is itself a count. Let $C$ be the predicate 'ARE-A-COUNT', defined as follows.

$$
\text { COUNT } C(x x) \equiv_{\mathrm{df}} x x \approx \top
$$

The fact that $x x$ must be interpreted by the same partition tells us that $x x$ are pairwise disjoint. But since $x x \approx \top$ is true, we know that $\bigcup \overline{x x}=\bar{\top}$, in which case $x x$ cover the whole universe. Thus, $C$ is true of $x x$ whenever $x x$ are a way of counting the domain of atoms.

Composition, then, is MANY-ONE cross-count identity given by $\approx$. In other words, $x x$ compose $y$ iff $x x$ are parts of $y$ and $y$ is covered by $x x$. It is plain to see that some parts compose a whole whenever those parts and the whole are the same portion of reality counted as many and counted as one, respectively. Moreover, the composition as identity theorist can appeal to standard first-order identity, classical mereology, standard plural identity, and the standard plural 'are-among' and 'is-one-of' predicates, since $\approx$ and $\lesssim$ are straightforward generalizations of these notions.

\section{LEIBNIZ'S LAW}

We now turn to Lewis's DISCERNIBILITY CHALLENGE. Lewis writes:

[E]ven though the many and the one are the same portion of reality, and the character of that portion is given once and for all whether we take it as many or take it as one, still we do not really have a generalized principle of the indiscernibility of identicals. It does matter how you slice it - not to the character of what's described, of course, but to get the form of the description. What's true of the many is not exactly what is true of the one. $(1991,87)$

Lewis appears to be right. Consider again the example of the tiled floor. The tiles are identical to the black and white squares. But it is

\footnotetext{
22 The proof is in Appendix 2.
} 
true of the tiles that they are tiles, whereas the black or white squares are not tiles - they are merely composed of tiles. While it is true of the black and white squares that they are squares, those squares could be made up of triangular tiles, which obviously are not squares. It does matter how you slice it.

But if we do not have the indiscernibility of identicals, we do not really have identity. Compare Sider (2007):

Defenders of strong composition as identity must accept Leibniz's Law; to deny it would arouse suspicion that their use of 'is identical to' does not really express identity. (57)

In short, the challenge is as follows:

DISCERNIBILITY CHALLENGE Is there any way to make sense of an identity predicate that engenders widespread failures of Leibniz's Law?

That is, if the truth of $x x \approx y y$ does not imply that every predicate $P$ is such that $P(x x)$ is true if and only if $P(y y)$ is true, then how does ' $\approx$ ' express anything like identity?

I claim that the DISCERNIBILITY CHALLENGE can be met. The solution involves cover-based semantics for distributive, collective, and cumulative predication in plural logics that require no restrictions on the indiscernibility of identicals. I provide two possible semantics: an indexical approach, and a subvaluational approach. Both versions fully satisfy Leibniz's Law, indicating that the general identity relation expressed by $\approx$ is legitimately considered a form of identity.

Before delving into the semantics, a brief aside. Donald Baxter (1988, 1999) has long been viewed as the sole defender of strong composition as identity. Virtually every paper published on the topic cites him in this way. ${ }^{23}$ But, for the sake of clarity, according to the earlier conception of identity that characterization is mistake. Baxter's view, too, is a form of weak composition as identity as he restricts the indiscernibility of identicals. ${ }^{24}$ The theory advanced in section 2 owes a great deal to his original theory given in (1988). While Baxter might well appeal to the semantics for plural predi-

${ }^{23}$ For a start, see Lewis (1991, p. 84), Sider (2007, 55, footnote 12), and Varzi (2000, 285). The lone exception appears to be Yi (1999, endnote 13).

${ }^{24}$ For details, see (1999). 
cates considered in section 3.2, it appears he prefers not to characterize identity as essentially involving Leibniz's Law.

\subsection{Plural predication}

Plural logic (in particular plural reference) opens up new ways of predicating. Some plural predication is distributive, where $P(x x)$ is distributive iff the truth of $P(x x)$ implies the truth of $P\left(x_{i}\right)$ for each $x_{i}$ among $x x$. A sentence like 'Tom and Dick are tall' is true iff Tom is tall and Dick is tall. This kind of plural predication is reducible to singular predication.

While distributive plural predication can be reduced in this way, other kinds of plural predication cannot. Some plural predication is collective. $P(x x)$ is collective iff $P(x x)$ is true only of the plurality and not of the individuals. For example, consider a sentence like 'Tom, Dick, and Harry carried the piano', which is true because they carried the piano together; none of them was capable of doing so individually.

One might suggest that English predicates like carried the piano are ambiguous: there are two distinct predicates, one of which is collective $\left(\right.$ CARRIED $\left._{C}\right)$ and the other distributive $\left(\right.$ CARRIED $\left._{D}\right){ }^{25}$ While this proliferates homonymous predicates, it solves the difficulty in interpreting collective and distributive predication.

There is, however, another kind of plural predication, cumulative predication, where $P(x x)$ is cumulative when $P(x x)$ is true of neither the individual objects, nor the objects collectively, but only of certain 'sub-pluralities'. ${ }^{26}$ To clarify, consider an example sentence: 'Rogers, Hammerstein, and Hart wrote musicals'. Now, is this sentence true? Presumably, but the three men never wrote any musicals together. Nor did any of them write any individually. Rogers and Hammerstein wrote musicals together, and Rogers and Hart wrote musicals together. So, we might say the predicate wrote musicals is true of Rogers, Hammerstein, and Hart, but only with respect to certain ways of 'carving up' the plurality.

\footnotetext{
25 As does Rayo (2002, 446).

${ }^{26}$ This distinction was made early on by Landman (1989), following Link. Landman discusses both collective and distributive predication, in addition to what he called 'group' predication. Other authors have called it 'intermediary' predication.
} 
Are there different homonymous predicates for every possible way of carving up the plurality? Consider the sentence 'The soldiers hit all four targets'. And suppose 'the soldiers' refer to Tom, Dick, and Harry. Do we need distinct predicates distinguishing the case where Tom hit two targets and the others hit only one from the case where Dick hit two and the others each hit one, and so on? Proliferating predicates is not likely to strike many as a natural fix. Moreover, it appears not to validate some intuitive inferences using them. ${ }^{27}$

Many linguists and philosophers of language have rejected this proliferation approach and have taken to interpreting predicates to be true of some individuals with respect to a given cover. ${ }^{28}$ What exactly is a cover?

COVER A set $P$ of non-empty sets $p_{1} \ldots p_{n}$ is a cover of $A$ if $\cup p_{1} \ldots p_{n}=A^{29}$

In other words, covers are just like partitions, but without the disjointness requirement. How does this help? The distributive sentence 'Tom and Dick are tall' is true with respect to the cover $\{\{$ Tom $\},\{$ Dick $\}\}$. The collective sentence 'Tom, Dick, and Harry carried the piano' is true with respect to the cover $\{\{$ Tom, Dick, Harry $\}\}$. And the cumulative sentence 'Rogers, Hammerstein, and Hart wrote musicals' is true with respect to the cover $\{\{$ Rogers, Hammerstein $\},\{$ Rogers, Hart $\}\}$.

While the 'correct' cover-based semantics for plural predicates is still very much up for debate, I consider two options for semantic clauses: an INDEXICAL semantics, and a SUBVALUATIONAL semantics.

On an INDEXICAL semantics, sentences involving plural predication are true relative to a cover. That is, plural terms may be interpreted by any cover, and the truth or falsity of the sentence varies with the interpretation. Where $c$ is any cover, and $\overline{x x_{c}}$ is the denotation of $x x$ under that cover, and $\bar{P}$ is the extension of $P$ :

INDEXICAL $\quad P(x x)$ is true-under-c iff $\overline{x x_{c}}$ is in $\bar{P}$

27 For discussion of these failures in inference, see Oliver and Smiley (2008).

${ }^{28}$ For more on this approach to plural predication, see Gillon (1992) and Nicolas (2008). An excellent discussion of cover-based approaches can be found in Schwarzschild (1996).

${ }^{29}$ For our purposes, I interpret covers set-theoretically, but they may be done hyperplurally as well. See Nicolas and Linnebo (2008) on hyperplural covers. 


\section{0 | Aaron J. Cotnoir}

On this approach, covers are additional parameters like contexts, times, or worlds. The truth of a sentence depends directly on the choice of a cover, on whether that choice is appropriate to the denotation of the plural terms. One advantage of this approach is that the very same predicate may be distributive, collective, and cumulative; no proliferation required.

It is crucial to note that there is no difficulty with Leibniz's Law. Imagine we have it that $a a=b b$ is true according to the standard conception of plural identity. Imagine also that a predicate $P$ is true of $\overline{a a_{c}}$ where $c=\left\{\left\{a_{1}, a_{2}\right\}\right\}$ and $P$ is true of $\overline{b b_{c}}$ where $c=\left\{\left\{b_{1}, b_{2}\right\}\right\}$. In other words, imagine $\overline{a_{1}}=\overline{b_{1}}$ and $\overline{a_{2}}=\overline{b_{2}}$ and $P$ is interpreted collectively. Leibniz's Law yields that the truth of $a a=b b$ implies the truth of $P(a a) \leftrightarrow P(b b)$. This is satisfied so long as both $a a$ and $b b$ are interpreted under $c$. It may be noted that if we interpret $P(a a)$ via $\overline{a a_{c^{\prime}}}$ where $c^{\prime}=\left\{\left\{a_{1}\right\},\left\{a_{2}\right\}\right\}$ and keep $P(b b)$ interpreted by $c, P(a a)$ will be false and $P(b b)$ will be true. But this is not a counterexample any more than it would be if we interpreted $a a$ and $b b$ at different times or at different worlds. ${ }^{30}$ The upshot is that the indiscernibility of identicals holds; but covers introduce an intensional (opaque) context because they are indexical.

For those uneasy with an INDEXICAL approach, one could instead use a SUBVALUATIONAL approach to the semantics of plural predication.

SUBVALUATIONAL $P(x x)$ is true iff there is a cover $c$, such that $\overline{x x_{c}}$ is in $\bar{P}$

Likewise, $P(x x)$ is false whenever there is no such cover (thus, avoiding any true contradictions). This semantic clause is not indexical; the truth of $P(x x)$ is not relative to any parameters. This approach also does not proliferate homonymous predicates, as the same sentence may be true when used distributively, collectively, or cumulatively.

And again, there is no difficulty with Leibniz's Law. Imagine we have it that $a a=b b$ is true according to the standard conception of plural identity. Let $P$ be true of $\overline{a a_{c}}$ where $c=\left\{\left\{a_{1}, a_{2}\right\}\right\}$ as before.

\footnotetext{
30 Thanks to an anonymous referee for this suggestion.
} 
Since $a_{1}=b_{1}$ and $a_{2}=b_{2}$, we know that there is at least one covernamely $c$-making $P(b b)$ true.

\subsection{Covers and counts}

We can now address the DISCERNIBILITY CHALLENGE by showing how $\approx$ fully satisfies the indiscernibility of identicals. The composition as identity theorist is in exactly the same situation as those giving semantics for plural predication. General identity given by $\approx$ is insensitive to our ways of carving up the domain; the identity of portions of reality is independent of how we count them. Other predicates are sensitive to these carvings. Parthood relations, for the defender of composition as identity, are expressed by predicates sensitive to counts. A singular individual is part of another singular individual only with respect to two different ways of countingone that treats the part as a singular thing, and another that treats the whole as a singular thing. The ONE-ONE version of $\lesssim$ is always cross-count.

Instead of covers, however, we model this phenomenon using partitions. Recall that partitions are simply covers plus a disjointness requirement. In the case of composition, there is independent reason for the disjointness requirement; Don Baxter (1988) and Achille Varzi (2000) argue that for ontological purposes, we ought to avoid 'double counting' certain portions of reality. The composition as identity theorist endorses the 'no double counting' requirement for ontological reasons, but the upshot for Leibniz's Law is no different here.

The composition as identity theorist can endorse the INDEXICAL semantics above: if $x x \approx y y$ is true-under- $c$ then every predicate $P$ (whether sensitive to counts or not) is such that $P(x x)$ is true-under-c if and only if $P(y y)$ is true-under-c. There are no failures of Leibniz's Law provided we do not switch our way of counting 'mid-sentence', as it were. Again, this is not a restriction on the indiscernibility of identicals; 'Socrates is sitting' could be true while 'Socrates is standing' false when interpreted at different times or different possible worlds. It is an interesting question as to why 'ways of counting' introduce intensional contexts. ${ }^{31}$ One possible answer: ways of

31 Thanks to Michael Della Rocca for alerting me to the importance of this question. 


\section{2 | Aaron J. Cotnoir}

counting are mind-dependent; they are ways of representing the world and not part of the world themselves. Perhaps then, they introduce intensional contexts in a similar way as 'believes that' does. But a full consideration of this question is beyond the scope of this chapter. For those who are not sympathetic to the INDEXICAL semantics, we turn to another option.

The composition as identity theorist can also endorse a SUBVALUATIONAL semantics; predicates are true of objects just if there is some way of counting the objects such that they have the property expressed by the predicate. Here again, $\approx$ can be shown to satisfy Leibniz's Law in full. Assume $a a \approx b b$ and $P(a a)$ are true. Then there is a count $c$ such that $\overline{a a_{c}}$ is in the extension of $P$. But since $\bigcup \overline{a a}=\bigcup \overline{b b}$, it follows that $\overline{b b_{c}}=\overline{a a_{c}}$. Hence, there is also a partition of $b b-$ namely $c$ - such that $\overline{b b} b_{c}$ is in the extension of $P$. Thus, $P(b b)$ is true.

Regardless of which semantics is preferred by the composition as identity theorist, I consider the DISCERNIBILITY CHALLENGE to be surmountable. The relation given by $\approx$ is a genuine identity relation.

Let me close this section with an interesting aside. (What remains of this section is tangential to the main arguments of this chapter and should be regarded as a more speculative line of thought for future research.) It is interesting to note that there may also be predicates $P_{\mathrm{i}}$ true of a portion of reality regardless of our way of counting. So, for these count-insensitive predicates, an additional SUPERVALUATIONAL semantic clause may be appropriate.

SUPERVALUATIONAL $P_{i}(x x)$ is true iff for any count $c, \overline{x x}_{c}$ is in $\bar{P}$

As in the case of predication in plural logic, this clause would be inappropriate for most predicates but perhaps appropriate for some. Of course, this new clause introduces no issues with Leibniz's Law for $\approx: P_{i}(a a)$ will be true exactly when $P(b b)$ is true given that $a a \approx b b$. Recall Lewis: 'It does matter how you slice it-not to the character of what's described, of course, but to get the form of the description' (italics added). The composition as identity theorist can utilize the SUBVALUATIONAL and SUPERVALUATIONAL semantics to explain Lewis's remark. The count-sensitive predicates like 'is a copse' and 'are five trees' are true in virtue of the form of our descriptions of the world; thus, they should receive the SUBVALUATIONAL semantics. On the other hand, there are count-insensitive predicates true of all ways of counting the copse or five trees: 'spatially 
extended', 'material', 'greenish', etc. These predicates are true in virtue of the character of the world; and should receive a SUPERVALUATIONAL semantics to reflect this difference.

\section{SIDER'S OBJECTIONS}

Sider (2007) has given some important objections to the thesis of composition as identity. ${ }^{32}$ In this section, I examine some of these objections and show how the current proposal avoids them. In each case, Sider's argument relies on collapsing the distinctions between ways of counting. Ways of counting preserve additional structure that is necessary for the logic of plurals to behave nicely, and Sider's arguments fail to go through in the presence of them.

\subsection{Collapse}

First, Sider shows that composition as identity (in conjunction with classical extensional mereology) implies the following principle.

COLLAPSE $y$ is part of the fusion of the $x x$ iff $y$ is one of $x x$

The principle says that $x x$ are all and only the parts of the fusion of $x x$. It arises from treating composition as numerical identity, rather than the general identity given by $\approx$. This principle causes some problems for the logic of plurals, and the composition as identity theorist ought to reject it. Consider again the tiled floor, where the black tiles are grouped into larger black squares and the white tiles are grouped into larger white squares. The fusion of the black squares and the white squares is the whole floor. But the black squares and white squares are not all of the parts of the floor; each individual tile is part of the floor, yet fails to be one of the black or white squares. So, the composition as identity theorist ought to reject the principle.

Sider's proof for COLLAPSE is as follows.

Left-to-right: let $a$ be the fusion of the $x x$, let $y$ be part of $a$. In addition to being composed of the $x x, a$ is composed of $y$ and $a$. Given strong compo-

${ }^{32}$ Early versions of at least one of these objections appeared in Yi (1999). Since Yi's objection relies on the same principles as Sider's, the main lines of my response to Sider will serve as a response to Yi. 


\section{4 | Aaron J. Cotnoir}

sition as identity, we get that $a=x x$, and also that $a=y$ and $a$; and so the $x x=y$ and $a$. Since $y$ is one of $y$ and $a$, it follows that $y$ is one of the $x x$. Right-to-left follows immediately from the definition of 'fusion'. 2007, 57-8)

The argument fails to apply to my proposal. To see why, let us follow along. Suppose $a$ is the fusion of $x x$. According to composition as identity, $a$ is the fusion of $x x$ iff $a$ is identical to $x x$. In our notation, $a \approx x x$. Also, let $y$ be a part of $a$, so $y \lesssim a$. Since, $\approx$ expresses general identity, we do in fact have it that $x x$ is identical to $y$ in the sense of being the same portion of reality. It is also the case that $y$ is composed of $y$ and $a$, so $y \approx y+a$ where + expresses binary fusion. But we do not have it that $y \approx y, a$ (where $y, a$ is the plurality made up of $y$ and $a$ ). Here we meet up with the first problem with Sider's argument. The issue is that since $y$ and $a$ are not disjoint, they cannot be among the same plurality. Recall, the plural terms have as referents members of the same partition. So, expressing $y \approx y$, $a$ is ruled out by the semantics.

No matter, though. As Sider himself notes, the argument can be run using $a \approx y, b$ where $b$ is just the mereological difference between $y$ and $a$ (this assumes that $y$ is a proper part of $a$ ). ${ }^{33}$ So far, then, we have it that $a \approx x x, y \precsim a$, and $a \approx y, b$. It does indeed follow (from the transitivity of $\approx$ ) that $x x \approx y, b$. This is an instance where $\approx$ is MANYMANY. As noted in section 2, however, MANY-MANY is not the same relation as plural identity. If it were, then since $y$ is one of $y, b$, it would follow that $y$ is one of $x x$. But it is not, and hence we do not necessarily have it that $y$ is one of $x x$.

Another way to see this point is by considering that the ONEMANY version $\lesssim$ expresses the plural 'is-one-of' if the terms are interpreted by the same partition. It only makes sense to say that one thing 'is-one-of' some other things if we are speaking according to a single way of counting. In Sider's proof, it would not follow that $y$ is one of $x x$ unless $y \sim x x$ is true. It is, however, worth noting that we do have it that $y \precsim x x$, even if they are interpreted via different counts. But in this case $\lesssim$ does not express the standard plural predicate.

This leads us to a related point of Sider's. He claims that COLLAPSE implies the failure of LISTS.

${ }^{33}$ See Sider (2007, 57, fn. 21). 
LISTS $x$ is one of $y y$ iff $x=y_{1}$ or... or $x=y_{n}$ (where each $y_{\mathrm{i}}$ is among $y y$ )

The failure of lists would be detrimental to plural logic. However, since on my account we do not have COLLAPSE, we may retain LISTS. In fact, we do have LISTs when $\lesssim$ expresses the 'is-one-of' relation (when $x \sim y y$ ). For in that case, $x \lesssim y y$ is true iff $\bar{x} \subseteq \bigcup \overline{y y}$. But given that partitions are pairwise disjoint, this can only happen if $\bar{x}=\bar{y}_{1}$ or ... or $\bar{x}=\bar{y}_{n}$ (where each $y_{\mathrm{i}}$ is among $y y$ ).

The main issue here is that Sider is using the plural 'is-one-of' predicate and the plural identity predicate across different ways of counting. This can seem legitimate on a theory according to which composition is just the standard numerical identity. But on my account, where composition is general identity, 'is-one-of' is strictly an intra-count notion that depends on the way one is counting the domain.

\subsection{Plural predication again}

Sider's second argument is that collective and distributive predication gets messed up. According to Sider:

There is no predicate carried the basket such that 'Tom, Dick, and Harry carried the basket' is true while 'Tick, Darry, and Hom carried the basket' is false where Tick = Tom's head + Dick's body (Dick minus his head), Darry $=$ Dick's head + Harry's body, and Hom $=$ Harry's head + Tom's body. For strong composition as identity implies that Tom, Dick, and Harry are identical to Tick, Darry, and Hom. $(2007,58)$

This argument is illuminating because it clearly shows how composition as numerical identity forces the collapse of distinct counts. While it is true that Tom, Dick, Harry are identical (MANY-MANY $\approx$ ) to Tick, Hom, Darry, they are explicitly different ways of counting the same portion of reality. On the INDEXICAL semantics, the truth of the sentence depends on the count used to interpret the plural term. So, on the Tom-Dick-Harry count, the sentence is true; on the Tick-Hom-Darry count, it is false. On the subvaluational semantics, 'Tick, Hom, and Darry carried the basket' is true, but only because there is a count which makes it true-namely, the collective count $\{\{$ Tom, Dick, Harry\}\}. 


\section{6 | Aaron J. Cotnoir}

The same holds true for distributive predication, which Sider claims the composition as identity theorist cannot have. Sider's argument goes like this:

Suppose $\Pi$ obeys Distribution, suppose $\Pi\left(x_{1}, \ldots, x_{n}\right)$, suppose $y_{1}+\ldots+y_{m}=x_{1}$ $+\ldots+\left({ }^{\prime}+\right.$ ' denotes fusion), and suppose $\neg \Pi\left(y_{i}\right)$ for some $i$. By strong composition as identity, we have $x_{1}, \ldots, x_{n}=x_{1}+\ldots+x_{n}$ and $y_{1} \ldots y_{m}=y_{1}+\ldots+y_{m}$; by transitivity and symmetry of identity, we have $x_{1}, \ldots, x_{n}=y_{1} y_{m}$; by Leibniz's Law, we have $\Pi\left(y_{1}, \ldots, y_{m}\right)$; by Distribution, we then have $\Pi\left(y_{i}\right)$; contradiction. $(2007,58)$

Everything in this argument is correct. But on the INDEXICAL view, the appeal to Leibniz's Law is illegitimate. If, under the supposition, $\Pi$ is true of $x_{1}, \ldots, x_{n}$ but not true of $y_{i}$ where $y_{i}$ is part of the fusion of $x_{1}, \ldots, x_{n}$, then clearly $\Pi$ is a count-sensitive predicate. As such, Leibniz's Law will only hold of plural terms interpreted under the same partition. However, by supposition $x_{1}, \ldots, x_{n}$ and $y_{1}, \ldots, y_{m}$ must be interpreted via different partitions. So, the argument does not go through.

On the subvaluational semantics, whether a predicate distributes or not depends entirely on partitions of the denotations of the plural terms. We can derive $\Pi\left(y_{1}, \ldots, y_{m}\right)$ since there is a partition of $y_{1}, \ldots, y_{m}$-namely, $\left\{\left\{x_{1}\right\}, \ldots,\left\{x_{n}\right\}\right\}$-according to which it is true. But we may not infer that $\Pi\left(y_{\mathrm{i}}\right)$, since $\Pi$ is distributive only relative to the $x$-partition and not the $y$-partition, by supposition.

So, it may well be that these considerations lead one to favour the INDEXICAL semantics over the SUBVALUATIONAL semantics (or vice versa). But, by no means do they cause problems for the logic of plurals on my theory.

Sider writes:

What underlies all these cases of unexpected behavior of plural expressions is that plural expressions are never 'tied to a unique decomposition' given strong composition as identity. $(2007,59)$

But on my proposal, plural expressions only ever refer to a portion of reality under a way of counting, and hence are tied to a unique decomposition. Sider is working with a theory of composition as numerical identity, which is forced to collapse this additional structure. By appealing explicitly to counts, composition as general identity can preserve this structure.

Moreover, it is not as if these 'ways of counting' are ad hoc fixes which 'tack on' additional structure to the theory in order to save it 
from Sider's objections. Ways of counting were an essential ingredient of composition as identity from its inception. Baxter's original idea, crucially, relied on ways of counting. ${ }^{34}$

I hope that the formalization given in this chapter will show that composition as identity is not as incoherent as has previously been argued. Moreover, it has significant advantages over Sider's version. I have given responses to Lewis's obstacles: the SYNTACTIC CHALLENGE, the SEMANTIC CHALLENGE, and the DISCERNIBILITY CHALLENGE. I have shown how to make sense of composition as identity in a way that avoids the strongest recent objections. The key to the problem is recognizing that the correct generalization of identity is a count-insensitive notion, that parthood and composition are cross-count notions, and that ordinary notions like identity, plural identity, and 'is-one-of' are intra-count notions.

The composition as identity theorist is free to endorse a single way of counting as the correct one, and in so doing would give an answer to the special composition question. And that answer need not be a universalist answer. ${ }^{35}$ Other coarser or more fine-grained counts would merely be 'loose talk' ${ }^{36}$ But composition as identity theorists are also free to claim that all ways of counting are equally good, as is assumed in Appendices 1 and 2. There are also intermediate views according to which some but not all ways of counting are correct. Whether 'ways of counting' themselves are objects which require additional ontological commitments is an interesting question. A negative answer is required in order for composition as identity to truly be ontologically innocent. But that will have to wait for another paper at some other time.

${ }^{34}$ See Baxter (1988). Wallace (2011a, 2011b) has attempted to use Baxter's notion of 'counts' to help composition as identity. She relativizes numerical identity to a way of counting, but does not give a generalized notion of identity. But it is unclear to me how composition (a cross-count notion) could be numerical identity (an intra-count) notion unless one has a general notion connecting the two. Moreover, Bøhn (2009) relativized identity to 'concepts', which behave somewhat like counts.

${ }^{35}$ Many authors have recently come to the conclusion that the thesis of composition as identity is independent of the special composition question. I think this is correct, because the thesis of composition as identity does not commit one to any particular ways of counting. See Cameron (2012), Effingham (MS), and McDaniel (2010) for more on this topic.

${ }^{36}$ See Baxter (1988). 


\section{8 | Aaron J. Cotnoir}

\section{APPENDIX 1: THE LANGUAGE LCI}

In this appendix, I provide a syntax and set-theoretic semantics for the language of composition as identity $L_{c i}$.

(a) Syntax for $L_{c I}$

\section{(i) Vocabulary}

Our formulas and sentences are built from a countable vocabulary:

- A set, $S C$, of singular constants: $\{a, b, c, \ldots\}$

- A set, $S V$, of singular variables: $\{x, y, z, \ldots\}$

- A set, $P C$, of plural constants: $\{a a, b b, c c, \ldots\}$

- A set, $P V$, of plural variables: $\{x x, y y, z z, \ldots\}$

- A set, $L P$, of logical predicates (binary): ${ }^{37}\{\precsim, \approx, \sim\}$

- A set, $Q$, of quantifiers: ${ }^{38}\left\{\forall_{1}, \forall_{2}, \exists_{1}, \exists_{2}\right\}$

- A set, $C$, of connectives (usual arity): ${ }^{39}\{\neg, \wedge\}$

- A set, $P$, of punctuation marks: $\{()$,

Constants in $S C$ or $P C$ and variables in $S V$ or $P V$ are terms.

\section{(ii) Formulas and Sentences}

We define our set of formulas, Form, recursively.

- For terms ss and $t t$, $(s s \precsim t t)$ and $(s s \approx t t)$ and $(s s \sim t t)$ are in Form. ${ }^{40}$

- If $\varphi \in$ Form and $v \in S V$, then $\forall_{1}(\varphi) \in$ Form and $\exists_{1} v(\varphi)$ Form.

- If $\varphi \in$ Form and $v v \in P V$, then $\forall_{2} v v(\varphi) \in$ Form and $\exists_{2} v v(\varphi) \in$ Form.

- If $\varphi \in$ Form then $\neg \varphi \in$ Form.

- If $\varphi, \psi \in$ Form then $(\varphi \wedge \psi) \in$ Form.

- Nothing else is in Form.

Our set of sentences, Sent $\subseteq$ Form, is merely the standard restriction of Form to those formulas where all occurring variables (if any) are bound. In the case of singular variables in $S V$, they must be bound

\footnotetext{
${ }^{37}$ Alternatively, we could treat $s \approx t$ as defined by $s \lesssim t \wedge t \lesssim s$.

${ }^{38}$ Alternatively, we could treat $\exists_{1} v(\varphi)$ and $\exists_{2} v v(\varphi)$ as defined by $\neg \forall_{1} v(\neg \varphi)$ and $\neg \forall_{2} v v(\neg \varphi)$.

${ }^{2}$ Treat $\supset, \vee, \equiv$ as defined in the usual way.

${ }^{40} \lesssim$ and $\approx$ and $\sim$ are polymorphic, taking either singular or plural terms.
} 
by $\forall_{1}$ or $\exists_{1}$; and in the case of plural variables in $P V$, they must be bound by $\forall_{2}$ or $\exists_{2}$.

(b) Semantics for $L_{C I}$

(i) Models

A model $M$, is a triple $\langle A, \Pi, I\rangle$ where $A \neq \varnothing$ (our atoms). $\Pi$ is the set of all partitions on $A$. $I$ is our interpretation function, which must satisfy the following.

- For all $c \in S C, I(c) \subseteq \pi$ for some $\pi \in \Pi$.

- For all $c c \in P C, I(c c) \subseteq \pi$ for some $\pi \in \Pi$.

\section{(ii) Variable Assignments}

A variable assignment, $i$, is a function that satisfies the following.

- For all $v \in S V, i(v) \subseteq \pi$ for some $\pi \in \Pi$.

- For all $v v \in P V, i(v v) \subseteq \pi$ for some $\pi \in \Pi$.

\section{(iii) Denotation}

We can now define a denotation function, $\delta$, s.t. for any term $t$ :

\section{(iv) Satisfaction}

$$
\delta(t)=\left\{\begin{array}{l}
I(t) \text { if } t \in S C \cup P C \\
i(t) \text { if } t \in S V \cup P V
\end{array}\right.
$$

A model and variable assignment satisfy a formula-written $M, i$ ₹ $\phi$-if and only if the following hold:

- For all terms $s s$ and $t t, M, i \vDash(s s \precsim t t)$ iff $\bigcup \delta(s s) \subseteq \bigcup \delta(t t)$.

- For all terms ss and $t t, M, i \vDash(s s \approx t t)$ iff $\bigcup \delta(s s)=\bigcup \delta(t t)$.

- For all terms $s s$ and $t t, M, i \vDash(s s \sim t t)$ iff there is some $\pi \in \Pi$, s.t. $\delta(s s)$ $\subseteq \pi$ and $\delta(t t) \subseteq \pi$.

- For all $v \in S V$ and $\varphi \in$ Form, $M, i \vDash \forall_{1} v(\varphi)$ iff $M, i^{\prime} \vDash \varphi$ for every $i^{\prime}$ that disagrees with $i$ only wrt $v$ (if at all); and $M, i \vDash \exists_{1} v(\varphi)$ iff $M, i^{\prime}$ $\vDash \varphi$ for some $i^{\prime}$ that disagrees with $i$ only wrt $v$ (if at all). 
- For all $v v \in P V$ and $\varphi \in$ Form, $M, i \vDash \forall_{2} v v(\varphi)$ iff $M, i^{\prime} \vDash \varphi$ for every $i^{\prime}$ that disagrees with $i$ only wrt $v v$ (if at all); and $M, i \vDash \exists_{2} v v(\phi)$ iff $M, i^{\prime} \vDash \phi$ for some $i^{\prime}$ that disagrees with $i$ only wrt $v v$ (if at all).

- For all $\varphi \in$ Form, $M, i \vDash \neg \varphi$ iff $M, i \not \models \varphi$.

- For all $\varphi, \psi \in$ Form, $M, i \vDash(\varphi \wedge \psi)$ iff $M, i \vDash \varphi$ and $M, i \vDash \psi$.

We say a model satisfies a formula-written $M \vDash \varphi$-iff $M, i \vDash \varphi$ for all $i$. We say a model and variable assignment satisfies $\Gamma \subseteq$ Formwritten $M, i \vDash \Gamma$-iff $M, i \vDash \gamma$ for all $\gamma \in \Gamma$.

\section{(v) Validity}

The argument from $\Gamma$ to $\varphi$ is valid iff for every $M$ and $i$, if $M, i \vDash \Gamma$, then $M, i \vDash \varphi$. A formula, $\varphi$, is valid iff for every $M, M \vDash \varphi$.

\section{APPENDIX 2: MEREOLOGY FOR}

In this appendix, I prove that the ONE-ONE version of $\lesssim$ satisfies the axioms for parthood in classical mereology.

Theorem. $\vDash \forall_{1} x(x \lesssim x) ; \vDash \forall_{1} x \forall_{1} y(x \lesssim y \wedge y \lesssim x \rightarrow x \approx y) ; \vDash \forall_{1} x$ $\forall_{1} y \forall_{1} z(x \lesssim y \wedge y \lesssim z \rightarrow x \lesssim z$.

Proof. These claims follow from the fact that $M, i \vDash s \lesssim t$ iff $\delta(s) \subseteq \delta$ $(t)$, and that $\subseteq$ is a partial order on $\wp(A)$.

Theorem. Strong Supplementation: $\vDash \forall_{1} x \forall_{1} y(x \nless d y) \rightarrow \exists_{1} z(z \lesssim x$ $\wedge \neg z \circ y)$

Proof. Fix an $M$, i s.t. $M, i \vDash \forall x \forall y(x \not y)$. So then $\delta(x) \nsubseteq \delta(y)$ under $i$. Now let $\delta(z)=\delta(x) \backslash \delta(y)$, and fix $i^{\prime}$ as the variable assignment that disagrees with $i$ wrt $z$ (if at all) by assigning $z$ to $\delta(x) \backslash \delta(y)$. Notice $\delta(z) \subseteq \delta(x)$ and for every $w \in \delta(z), w \notin \delta(y)$. Hence, $M, i^{\prime} \vDash z \lesssim x \wedge$ $\ulcorner z \circ y)$ and so $M, i \vDash z \lesssim x \wedge \neg z \circ y)$. $i^{\prime}$ is a legitimate variable assignment since every subset of our domain $A$ of atoms is a member of some possible partition on $A$. Since $\delta(z) \subseteq A$ and $\Pi$ is the set of all possible partitions, $\delta(z) \in \pi$ for some $\pi \in \Pi$.

Theorem. Plural Unrestricted Fusion: $\vDash \forall_{2} x x \exists_{1} y \forall_{1} z(z \circ y \leftrightarrow$ $\left.\exists_{1} x(x \lesssim x x \wedge z \circ x)\right)^{41}$

${ }^{41}$ Here $x \circ y$ is an abbreviation for $\exists_{1} w(w \lesssim x \wedge w \lesssim y)$. 
Proof. Let $\delta(x x) \subseteq \pi$ where $\pi \in \Pi$ ( $\pi$ is a partition on $A$ ). Now fix $\delta(y)=\bigcup \delta(x x)$. Notice that, in the set-theoretic interpretation, $a \circ b$ iff $\delta(a) \cap \delta(b) \neq \varnothing$. Since $\delta(z) \in \varnothing(A)$, we know that $\delta(z) \cap \delta(y) \neq \varnothing$ iff $\delta(z) \cap \bigcup \delta(x x) \neq \varnothing$. By simple set theory, then, $\delta(z) \cap \delta\left(x_{i}\right) \neq \varnothing$ for some $x_{i}$ among $x x .^{42}$

University of St Andrews

\section{REFERENCES}

Baxter, D. Identity in the loose and popular sense. Mind 97 (1988a), 576-82.

Baxter, D. Many-one identity. Philosophical Papers 17 (1988b), 193-216.

Baxter, D. The discernibility of identicals. Journal of Philosophical Research 24 (1999), 37-55.

Bøhn, E. Composition as Identity. PhD thesis, University of Massachusetts, 2009. <http://scholarworks.umass.edu/open_access_dissertations/92/>.

Cameron, R. Composition as identity doesn't settle the special composition question. Philosophy and Phenomenological Research 85, 3 (2012), 531-54.

Effingham, N. Debunking a mereological myth: if composition as identity is true, then universalism need not be. Unpublished manuscript: <http:/ / www.nikkeffingham.com/ resources / Debunking+a+ Mereological+Myth.pdf $>$.

Frege, G. The Foundations Of Arithmetic: A Logico-Mathematical Enquiry into the Concept of Number, 2nd edn. Evanston, IL: Northwestern University Press, 1980.

Gillon, B. Towards a common semantics for English count and mass nouns. Linguistics and Philosophy 15, 6 (1992), 597-639.

Landman, F. Groups I. Linguistics and Philosophy 12, 5 (1989), 559-605.

Laycock, H. Words without Objects. Oxford: Clarendon Press, 2006.

Lewis, D. Parts of Classes. Oxford: Basil Blackwell, 1991.

${ }^{42}$ This chapter arose out of two excellent seminars I took while a graduate student at the University of Connecticut: Marcus Rossberg's seminar on higher-order and plural logics, and Don Baxter's seminar on identity in metaphysics. Thanks to the following for many helpful discussions on these issues: Andrew Bacon, Ralf Bader, Jc Beall, Don Baxter, Einar Bøhn, Colin Caret, Michael Della Rocca, Tim Elder, Tamar Gendler, Katherine Hawley, Michael Hughes, Philip Kremer, Jon Litland, Doug Owings, David Nicholas, Agustín Rayo, Marcus Rossberg, Yael Sharvit, Zoltan Szabo, John Troyer, Gabriel Uzquiano, Achille Varzi, Sam Wheeler, Bruno Whittle, and Jessica Wilson. Finally, I wish to thank four anonymous referees for comments that led to many improvements of this chapter. 
McDaniel, K. Composition as identity does not entail universalism. Erkenntnis 73, 1 (2010), 97-100.

Nicolas, D. Mass nouns and plural logic. Linguistics and Philosophy 31, 2 (2008), 211-44.

Nicolas, D., and Linnebo, O. Superplurals in English. Analysis 68, 3 (2008), 186-97.

Oliver, A., and Smiley, T. Is plural denotation collective? Analysis 68, 1 (January 2008), 22-34.

Rayo, A. Word and objects. Noûs 36, 3 (2002), 436-64.

Rayo, A. Beyond plurals. In A. Rayo and G. Uzquiano (eds), Absolute Generality. Oxford: Oxford University Press, 2006, pp. 220-54.

Saucedo, R. Composition, identity, and the number of things. Unpublished manuscript.

Schlenker, P. Properties, plurals, and paradox. Unpublished manuscript: <http://www.linguistics.ucla.edu/people/schlenker/PPP.pdf>.

Schwarzschild, R. Pluralities. Dordrecht: Kluwer, 1996.

Sharvey, R. A more general theory of definite descriptions. The Philosophical Review 89 (1980), 607-24.

Sider, T. Parthood. The Philosophical Review 116 (2007), 51-91.

Uzquiano, G. The supreme court and the supreme court justices: A metaphysical puzzle. Noûs 38, 1 (2007), 135-53.

van Inwagen, P. Composition as identity. In J. Tomberlin (ed.), Philosophical Perspectives, vol. 8. Ridgeview, Atascadero CA, 1994, pp. 207-20.

Varzi, A. Mereological commitments. Dialectica 54 (2000), 283-305.

Wallace, M. Composition as identity: Part I. Philosophy Compass 6 (2011a), 804-16.

Wallace, M. Composition as identity: Part II. Philosophy Compass 6 (2011b), 817-27.

Yi, B. Is mereology ontologically innocent? Philosophical Studies 93 (1999), 141-60. 